\title{
Can the combination of a negative computed tomography result and a negative lumbar puncture safely exclude the diagnosis of subarachnoid hemorrhage in patients with thunderclap headache?
}

\author{
Reviewed by: Mohammed Aref Malabarey, MD*; David Barbic, MD, MSc*
}

Clinical question

How many patients who had a negative computed tomography and lumbar puncture result on initial evaluation were diagnosed with subarachnoid hemorrhage in the subsequent 6 months to 3 years?

Article chosen

Perry J, Spacek A, Forbes M, et al. Is the combination of negative computed tomography result and negative lumbar puncture result sufficient to rule out subarachnoid hemorrhage? Ann Emerg Med 2008;51:707-13.

Objective

To determine the sensitivity of both a negative computed tomography and a negative lumbar puncture in ruling out subarachnoid hemorrhage.

Keywords: computed tomography, lumbar puncture, subaforachnoid hemorrhage

\section{BACKGROUND}

Thunderclap headache is an acute and severe headache that attains its maximum intensity within 1 hour of

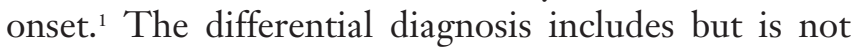
limited to subarachnoid hemorrhage (SAH), cerebral venous sinus thrombosis, cervical artery dissection, and pituitary apoplexy. ${ }^{2-4}$ Current clinical practice and major print and online emergency medicine textbooks suggest that a negative computed tomographic (CT) head scan combined with a negative lumbar puncture (LP) result are adequate to rule out $\mathrm{SAH}$ in patients with acute headache presenting to the emergency department (ED). ${ }^{5-8}$ Several studies support this practice; however, all of these studies suffered from a small sample size $(71,14,18,137$, and 93 patients, respectively; $N=333) .{ }^{9-13}$ These studies used followup ranging from 12 months to 3.3 years as their reference standard. During the follow-up period, no patients were identified with SAH.

\section{POPULATION STUDIED}

Investigators enrolled consecutive patients, 15 years of age and older, presenting to the ED with acute headache. Inclusion criteria included "headache patients who had the following characteristics: alert, defined by a Glasgow Coma Scale score of 15 (i.e., awake and fully oriented); nontraumatic, defined as no falls or direct trauma to the head in the previous 7 days; and acute, defined as reaching maximal intensity in less than 1 hour and the patient presenting to the ED within 14 days of the headache's onset." 1

Patients were excluded if they fell into one of the following categories: 1) recurrent headaches, that is, three or more headaches of the same character and severity during a period of greater than 6 months, (i.e., a likely diagnosis of migraine headaches); 2) confirmed $\mathrm{SAH}$, that is, by CT or lumbar puncture before presentation at the study ED; 3) reassessment of the same headache, that is, previously investigated with

From the *McGill Emergency Medicine Residency Program, Royal Victoria Hospital, Montreal, QC.

Correspondence to: Dr. David Barbic, McGill Emergency Medicine Residency Program, Royal Victoria Hospital, 687 Pine Avenue, Montreal, QC H3A 1A1; david.barbic@mail.mcgill.ca.

This article has been peer reviewed.

(C) Canadian Association of Emergency Physicians

CJEM 2012;15(2):113-115

DOI $10.2310 / 8000.2012 .120850$

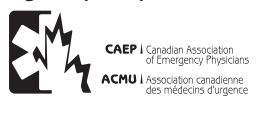

$C J E M \cdot J C M U$

2012;15(2) 
both CT and lumbar puncture for the same headache; 4) papilledema on examination; 5) new focal neurologic deficit on physical examination; 6) previous diagnosis of SAH; 7) previous diagnosis of a brain neoplasm; 8) neurologic shunt; and 9) post-LP headache, that is, headache within 72 hours after an LP.

\section{STUDY DESIGN}

The authors employed a prospective cohort study at two tertiary care Canadian academic EDs for patients with the above-mentioned inclusion criteria. Patients were followed up for a period of time ranging from 6 months to 3 years to identify those who developed SAH during the follow-up. The follow-up consisted of a telephone questionnaire determining whether or not the patient developed a subsequent SAH. In addition to the telephone call, investigators also searched the electronic computer system at the study hospitals to determine whether patients developed an SAH after their initial ED visit.

\section{OUTCOMES MEASURED}

The primary outcome was SAH, "which was defined by one of the following: (1) subarachnoid blood on CT, as reported in the final neuroradiology report; (2) xanthochromia in the cerebrospinal fluid by visual inspection of the centrifuged supernatant; (3) [red blood cells $(\mathrm{RBCs})]$ in the final tube of cerebrospinal fluid $(>5 \times$ $10^{6} \mathrm{RBCs} / \mathrm{L}$ ), with an aneurysm demonstrated on cerebral angiography (traditional, CT, or magnetic resonance imaging $[\mathrm{MRI}]$ ); or (4) autopsy results confirming subarachnoid hemorrhage." Patients included in the study were contacted by telephone as a follow-up for verifying whether they did or did not develop any subsequent SAH using the questionnaire.

Patients were considered negative for SAH if they had not had an SAH subsequent to their initial ED visit.

\section{RESULTS}

Of the 592 patients enrolled in the study, 61 patients had SAH during the study period; $89.6 \%$ of patients were followed up: $80.4 \%$ by telephone and the remaining $9.2 \%$ through their visits to the hospital.

None of the patients who were followed up had a subsequent SAH. One patient, however, was found to have cerebral aneurysms on cerebral angiography, although this patient never developed SAH; the cerebral angiography was done as a screening test due to the strong family history of cerebral aneurysms.

The authors included a primary analysis and a sensitivity analysis in their article. Primary and sensitivity analyses were demonstrated in tables. The primary analysis assumed that none of the patients lost to followup had SAH, in which the following results were obtained: the strategy (negative CT and negative LP) showed a sensitivity of $100 \%$ (95\% CI 94-100), a specificity of $67 \%$ (95\% CI 63-71), a negative predictive value of $100 \%$, and a negative likelihood ratio < 0.00001 . In the secondary analysis, the authors assumed that one of the patients lost to follow-up did have a missed SAH, and the following results were obtained: the strategy showed a sensitivity of $98 \%$ (95\% CI 91$100)$, a specificity of $67 \%$ (95\% CI $63-71 \%$ ), a negative predictive value of $99.7 \%$, and a negative likelihood ratio of 0.024 (J. Perry, personal communication, 2011).

The second sensitivity analysis, which was not demonstrated in the tables, assumed that one of the patients lost to follow-up had an SAH, which showed the following results: the strategy showed a sensitivity of $97 \%$ (95\% CI 89-99) and a negative likelihood ratio of 0.047 (95\% CI $0.01-0.17)$.

\section{COMMENTARY}

Of patients presenting to the ED with an acute headache, SAH accounts for only $1 \%$ of these patients, yet SAH has a prevalence of $12 \%$ for true thunderclap headaches. ${ }^{12}$ One in 20 cases of SAH is missed, with a mortality rate exceeding $50 \%$ in the first 30 days if untreated..$^{14}$

Almost all of the studies examining this topic to date were retrospective. Most of them were consistent with the conclusion that a negative CT result on its own is insufficiently sensitive to rule out such a life-threatening condition. ${ }^{9-13}$ These five studies examined a total of 333 patients mixed between prospective and retrospective study designs, with follow-up ranging from 12 months to 3.3 years. The sensitivity of CT to rule out $\mathrm{SAH}$ in these studies varied from 80 to $90.1 \%$. These studies were all completed prior to 2002 and the more recent advances in CT technology. One recent study examining fifth-generation CT scanners showed a sensitivity and a specificity of $100 \%$ and $99.4 \%$, respectively, for the diagnosis of SAH..$^{15}$

The literature indicates that the strategy of negative CT and negative LP is safe to rule out SAH..$^{9-13,16,17}$ However, the major limitation of the study we reviewed is the number of patients who were lost to follow-up $(n=60)$. 


\begin{tabular}{|c|c|c|}
\hline \multirow[b]{2}{*}{ Strategy } & \multicolumn{2}{|c|}{ SAH } \\
\hline & Yes & No \\
\hline Positive & 61 & 175 \\
\hline Negative & 7 & 349 \\
\hline \multicolumn{3}{|c|}{$\begin{array}{l}\text { SAH = subarachnoid hemorrhage. } \\
\text { Sensitivity }=61 / 61+7=.92=89.7 \% \text {; specificity }=349 / 349+175=.667=66.6 \% \\
\text { negative likelihood ratio }=(1-0.897) / 0.666=0.15 .\end{array}$} \\
\hline
\end{tabular}

In their sensitivity analysis, the authors assumed that one of the patients lost to follow-up did develop an SAH. The study strategy still showed a sensitivity of 97\% (95\% CI 89-99), with a negative likelihood ratio of 0.047 (95\% CI 0.01-0.17).

If we assume that 7 of the 60 patients (given the fact that the prevalence of SAH for thunderclap headaches $=12 \%)^{7}$ lost to follow-up did turn out to have an SAH and presented to a community hospital or to any other hospital outside the study province or even abroad, the results shown in Table 1 would have been obtained.

It should be noted that the authors of this study published further research on this topic in early 2011 to examine whether CT performed within 6 hours was sensitive to detect SAH..$^{18}$ A total of 3,132 patients were prospectively enrolled from 11 Canadian tertiary care EDs who presented with new-onset acute (thunderclap) headache. For the 953 patients scanned within 6 hours of headache onset, all 121 patients with SAH were identified by CT, yielding a sensitivity of $100 \%$ $(97.0-100.0 \%)$ and a specificity of $100 \%$ (99.5$100 \%) .{ }^{18}$ This is consistent with the findings of Boesiger and Shiber mentioned previously. ${ }^{15}$

\section{CONCLUSION}

The findings of this study seem to support the accepted teaching that negative CT and negative LP results are sensitive enough to rule out $\mathrm{SAH}$ in patients presenting with thunderclap headache. ${ }^{1}$ Given that recent studies have demonstrated sensitivities of $100 \%$ for ruling out SAH with newer-generation CT scanners, the accepted strategy for emergency physicians to rule out SAH may be shifting toward a negative CT-only paradigm.

Competing interests: None declared.

\section{REFERENCES}

1. Perry J, Spacek A, Forbes M, et al. Is the combination of negative computed tomography result and negative lumbar puncture result sufficient to rule out subarachnoid hemorrhage? Ann Emerg Med 2008;51:707-13, doi:10.1016/j.annemergmed.2007.10.025.

2. Schwedt TJ, Matharu MS, Dodick DW. Thunderclap headache. Lancet Neurol 2006;5:621-31, doi:10.1016/S14744422(06)70497-5.

3. Savitz SI, Edlow J. Thunderclap headache with normal CT and lumbar puncture: further investigations are unnecessary. Stroke 2008;39:1392-3, doi:10.1161/STROKEAHA.107.503151.

4. Savitz SI, Levitan EB, Wears R, et al. Pooled analysis of patients with thunderclap headache evaluated by CT and LP: is angiography necessary in patients with negative evaluations? f Neurol Sci 2009;276:123-5, doi:10.1016/ j.jns.2008.09.019.

5. Singer RJ, Ogilvy CS, Rordorf G. Etiology, clinical manifestations, and diagnosis of aneurysmal subarachnoid hemorrbage. Available at: http://www.uptodate.com (accessed September 13, 2011).

6. Becske T. Subarachnoid hemorrbage workup. Available at: http://emedicine.com (accessed September 13, 2011).

7. Tintinalli J, Stapczynski J, Ma OJ, et al. Tintinalli's emergency medicine: a comprehensive study guide. 7th ed. New York: McGraw-Hill Professional; 2010.

8. Marx JA, Hockberger RS, Walls RM, et al. Rosen's emergency medicine. Philadelphia: Mosby; 2010.

9. Wijdicks EF, Kerkhoff H, van Gijn J. Long-term follow-up of 71 patients with thunderclap headache mimicking subarachnoid haemorrhage. Lancet 1988;2:68-70, doi:10. 1016/S0140-6736(88)90004-9.

10. Harling D, Peatfield R, Hille P. Thunderclap headache: is it migraine? Cephalalgia 1989;9:87-90.

11. Markus H. A prospective follow up of thunderclap headache mimicking subarachnoid haemorrhage. 7 Neurol 1991;54: 1117-25.

12. Landtblom A, Fridriksson S, Boivie J. Sudden onset headache: a prospective study of features, incidence and causes. Cephalalgia 2002;22:354-60, doi:10.1046/j.1468-2982.2002. 00368.x.

13. Linn F, Rinkel G, Algra A. Follow-up of idiopathic thunderclap headache in general practice. 7 Neurol 1999; 246:946-8, doi:10.1007/s004150050488.

14. Leblanc R. The minor leak preceding subarachnoid hemorrhage. 7 Neurosurg 1987;66:35-9, doi:10.3171/jns.1987.66.1.0035.

15. Boesiger BM, Shiber JR. Subarachnoid hemorrhage diagnosis by computed tomography and lumbar puncture: are fifth generation CT scanners better at identifying subarachnoid hemorrhage? f Emerg Med 2005;29:23-7, doi:10.1016/ j.jemermed.2005.02.002.

16. MacDonald A, Mendelow AD. Xanthochromia revisited: a re-evaluation of lumbar puncture and CT scanning in the diagnosis of subarachnoid haemorrhage. 7 Neurol Neurosurg Psychiatry 1988;51:342-4, doi:10.1136/jnnp.51.3.342.

17. van der Wee N, Rinkel GJ, Hasan D, et al. Detection of subarachnoid haemorrhage on early CT: is lumbar puncture still needed after a negative scan? 7 Neurol Neurosurg Psychiatry 1995;58:357-9, doi:10.1136/jnnp.58.3.357.

18. Perry JJ, Stiell IG, Sivilotti MLA, et al. Sensitivity of computed tomography performed within six hours of onset of headache for diagnosis of subarachnoid haemorrhage: prospective cohort study. BMF 2011;343:d4277, doi:10.1136/ bmj.d4277. 